\title{
Quantitative measures of tissue mechanics to detect hypermobile Ehlers-Danlos syndrome and hypermobility syndrome disorders: a systematic review
}

\author{
Shea Palmer ${ }^{1}\left(\mathbb{D} \cdot\right.$ Elise Denner $^{1} \cdot{\text { Matthew } \text { Riglar }^{1} \cdot \text { Holly Scannell }^{1} \cdot \text { Sarah Webb }}^{1} \cdot$ Georgina Young $^{1}$
}

Received: 3 December 2019 / Revised: 6 January 2020 / Accepted: 10 January 2020 / Published online: 18 January 2020

(C) The Author(s) 2020

\begin{abstract}
Hypermobile Ehlers-Danlos syndrome (hEDS) and hypermobility spectrum disorders (HSD) are heritable connective tissue disorders associated with pain, activity limitations and participation restrictions. A key feature is reported to be reduced stiffness and increased extensibility and elasticity of connective tissues. Yet diagnosis relies on assessment of joint range of motion, which may be influenced by other factors, and semi-quantitative assessment of forearm skin extensibility. The objective of this systematic review was to determine if quantitative measures of tissue mechanics can discriminate between hEDS/HSD and healthy tissues. Literature was identified via online databases (AMED, CINAHL+, EMBASE, MEDLINE and SportDiscus) and snowballing. Studies were included if participants had a confirmed diagnosis of hEDS/HSD (or equivalent diagnosis) using internationally recognised criteria, a healthy control group was used as a comparator, and objective measures of tissue stiffness, extensibility or elasticity of muscle, tendon, connective tissue or skin were reported. Included studies were critically appraised, followed by group discussion, consensus and narrative synthesis. Two hundred three potentially relevant studies were identified. Application of the inclusion criteria resulted in four studies being included. A range of quantitative approaches to studying tissue mechanics were used, including diagnostic ultrasound. Overall, three of the four studies found that at least one measure of tissue mechanics distinguished between people with hEDS/HSD and healthy controls. The studies were generally conducted and reported to high standards. Quantitative measures of tissue mechanics have the potential to contribute towards more objective diagnosis of hEDS/HSD. Further validation, particularly within diagnostic scenarios, is required.
\end{abstract}

Keywords Connective tissue diseases $\cdot$ Ehlers-Danlos syndrome $\cdot$ Elasticity $\cdot$ Familial $\cdot$ Joint laxity

\section{Introduction}

Hypermobile Ehlers-Danlos syndrome (hEDS) [1] and hypermobility spectrum disorders (HSD) [2] are heritable connective tissue disorders that present with multiple hypermobile joints and pain. Both conditions may be associated with a variety of other manifestations affecting autonomic, cardiovascular and gastrointestinal function; however, these are more commonly found in the rarer sub-types of EDS [3].

Shea Palmer

shea.palmer@uwe.ac.uk

1 Department of Allied Health Professions, Faculty of Health \& Applied Sciences, University of the West of England, Blackberry Hill, Bristol BS16 1DD, UK
For example, there is a very high prevalence of self-reported orthostatic intolerance (98\% of respondents), easy bruising (97\%) and urinary incontinence (84\%) [4]. Further studies have reported a higher total number of injuries and a higher prevalence of sprains, dislocations, back pain, clumsiness, easy bruising and balance problems [5]; and detrimental effects on pain, depression, fatigue, sleep and general health [6] relative to healthy control participants.

In 2017, a new international nosology and diagnostic criteria for EDS were published [1], replacing the previous Villefranche nosology for EDS [7] and Brighton criteria for joint hypermobility syndrome (JHS) [8]. There are 13 EDS categories, 12 of which are rare and have specific genetic markers, each presenting with a different range of symptoms and severity. The 2017 and Villefranche nosologies both included a classification for which there is no identified genetic marker, namely hEDS and EDS-hypermobility type (EDSHT) (previously EDS type III) respectively. Patients who 
present with symptomatic joint hypermobility but do not meet the strict criteria for hEDS may now receive a diagnosis of HSD [2]. For the purpose of this study, and for ease of reporting, the terms hEDS/HSD will be used to refer to EDS-HT/JHS, except where authors employ specific diagnostic terms.

One of the signs of hEDS/HSD is increased tissue elasticity, and this is believed to have a negative impact on passive and active joint stability [9]. Joint instability may result from ligamentous laxity but also altered force production and transmission through the musculotendinous structures. Tissue elasticity is traditionally assessed in these conditions using the 9point Beighton score of joint motion [10]. A score of $\geq 4 / 9$ [8] or $\geq 5 / 9$ [7] was previously used as part of the diagnostic criteria to indicate generalised joint hypermobility. Agespecific cut-offs of $\geq 4, \geq 5$ or $\geq 6$ are now advocated [1]. However, one might question the validity of the Beighton score as an indicator of a specific connective tissue disorder because it does not include many commonly affected joints [11], does not assess connective tissues more widely and may be affected by stretching exercise, such as in dancers [12]. The Beighton score may be affected by age, sex and ethnicity [13], and theoretically by habitual postures and movements.

The 2017 criteria for hEDS does also include semiquantitative assessment of skin extensibility in the volar aspect of the forearm, with a value of $>1.5 \mathrm{~cm}$ considered positive [1]. The basis for this cut-off of $>1.5 \mathrm{~cm}$ is unclear, however, as the Villefranche criteria [7] did not provide a cut-off and the referenced source [14] only reported the inter-rater reliability of using a 2-cm cut-off for abnormality. Interestingly, agreement between examiners ranged from 0.44 to 0.72 , below the a priori acceptable level of 0.80 [14]. No other assessment of tissue mechanics is advocated as part of diagnosis.

These issues highlight the potential for improved objective assessment of tissue mechanics as part of hEDS/HSD diagnosis and a number of authors have previously attempted to investigate this issue. For example, Alsiri and colleagues [15] found that strain elastography differentiated between people with HSD and healthy controls in a range of muscles and tendons. Using ultrasonography, Rombaut and colleagues [16] demonstrated reduced Achilles tendon stiffness in EDSHT compared to controls during an isometric contraction. Heidbreder and colleagues [17] demonstrated that a simple suction cup tool could aid the diagnosis of classic-type EDS versus vascular-type EDS, spontaneous cervical artery dissection and a control group. Indeed, Grahame and Beighton [18] used the suction cup method to plot stress-strain curves for skin elasticity in EDS patients half a century ago. The evidence related to the potential of such objective methods to differentiate between hEDS/HSD and healthy tissues is currently unclear, however. A systematic approach to identifying and evaluating the evidence is required to understand the potential of such methods to improve the diagnostic process.
The objective of this systematic review was therefore to determine whether quantitative measures of tissue mechanics are able to distinguish between hEDS and healthy controls or HSD and healthy controls.

\section{Method}

This review is reported according to preferred reporting items for systematic reviews and meta-analyses (PRISMA) recommendations [19]. The review was not prospectively registered in the International Prospective Register of Systematic Reviews (PROSPERO).

\section{Search strategy and study selection}

A subject librarian with expertise in systematic reviewing advised on the selection of electronic databases and the search strategy. The electronic databases selected were AMED, CINAHL+, EMBASE, MEDLINE and SportDiscus. Search terms and associated synonyms (Table 1) were developed through group discussion and advice from the subject librarian.

The diagnostic terms of hEDS and HSD were included as well as previous diagnoses of JHS, EDS-HT and EhlersDanlos type III, as it was expected that limited literature would be retrieved using the $2017 \mathrm{hEDS}$ and HSD diagnostic terms $[1,2]$. The search was limited to the English language and the time period January 1, 1998 to January 31, 2019 because the Villefranche nosology and Brighton criteria were only in the public domain from 1998. Two researchers conducted the electronic database searches to minimise potential errors.

Inclusion criteria, agreed a priori through group discussion, were as follows: (1) confirmed diagnosis of hEDS/HSD (or equivalent diagnoses of EDS-HT, EDS type III or JHS) using internationally recognised criteria; (2) healthy control group as a comparator and (3) objective measures of tissue stiffness, extensibility or elasticity of muscle, tendon, connective tissue or skin. There were no limitations on study type, other than including only primary research.

All duplicates and non-human studies were removed. Study titles were then screened against the inclusion criteria at a meeting of all researchers. The abstracts of the remaining studies were all screened by individual team members, followed by another group meeting to agree which would be included for full-text review. Any disputes were discussed and resolved by consensus. Snowballing of the reference lists of remaining studies was undertaken to identify potential additional studies and these were screened in the same way. The remaining studies underwent full-text review by individual team members, with the final studies to be included in the review then agreed at a meeting of the research group. The 
Table 1 Electronic database search terms

S1 'Hypermobile EDS' OR 'hEDS' OR 'Hypermobility spectrum disorder' OR 'HSD' OR 'Ehlers-Danlos syndrome hypermobility type' OR

'EDS-HT' OR 'Joint hypermobility syndrome' OR 'JHS' OR 'Ehlers-Danlos Type III'

S2 'Measur*' OR 'Classif*' OR 'Quantif*' OR 'Monitor*' OR 'Estimat*'

S3 'Muscle' OR 'Tendon' OR 'Tissue' OR 'Skin'

S4 'Extens*' OR 'Elast*' OR 'Stiff*' OR 'Strain' OR 'Stretch' OR 'Laxity'

S5 S1 AND S2 AND S3 AND S4

search and selection processes were recorded as per PRISMA guidelines [19].

\section{Critical appraisal and data extraction}

The 'strengthening the reporting of observational studies in epidemiology' (STROBE) checklist [20] was chosen as a framework to facilitate critical appraisal in this review. Although the STROBE checklist is not a critical appraisal tool per se, as an international reporting standard, it addresses the key aspects of design, conduct and analysis of cross-sectional studies. As such, it was considered a valuable framework to judge the quality of the included studies. Each individual team member completed the STROBE checklist for each of the final included studies. Further information about each study was recorded using a standardised data extraction form. Data extracted included study design, country, aims, tissue(s) investigated, diagnostic criteria, sample size, sex, age, ethnicity, patient recruitment, statistical analysis and a final summary. A final version of the STROBE checklist and data extraction table for each study was then agreed through discussion and consensus.

\section{Results}

The PRISMA flow diagram is shown in Fig. 1.

A summary of the characteristics of each of the four studies can be seen in Table 2 and the STROBE statement outcomes can be found in Table 3 .

Table 2 identifies that four studies were completed across three countries. Total sample sizes ranged from 23 to 50, with 6 to 25 participants in the hEDS/HSD (or EDS-HT/JHS) groups. A much higher proportion of women than men were included. Evidence was presented for skin extensibility, skin elasticity, skin consistency, tendon stiffness, tendon resistive torque, tendon elasticity, tendon deformation, tendon strain, muscle strain and muscle elasticity. The anatomical sites tested included both the volar and dorsal aspects of the forearm [14]; the Achilles tendon, patellar tendon and five muscles [15]; the Achilles tendon [16] and the patellar tendon [21].

Overall, three of the four studies found that at least one measure of tissue mechanics distinguished between people with hEDS/HSD and healthy controls. These included skin consistency [14]; Achilles tendon stiffness [16]; upper boundary of the strain index of the brachioradialis muscle, patellar tendon and Achilles tendon [15]; strain ratio of the brachioradialis muscle and patellar tendon [15]; the proportion of 'soft' tissues in the biceps brachii muscle and Achilles tendon [15]; and the proportion of 'hard' tissues in the biceps brachii and brachioradialis muscles and the Achilles tendon [15]. The fourth study [21] was able to distinguish between classical EDS and controls but not between BJHS and controls.

Table 3 illustrates that the studies were generally well-reported, with additional information required for some studies for only a small number of criteria related to setting, bias, study size and funding. Alsiri and colleagues [15] was the only study to meet all STROBE criteria.

\section{Discussion}

Pertinent issues identified as part of the critical appraisal process will now be discussed within the context of existing literature.

\section{Study characteristics}

Table 2 summarises the characteristics of the included studies, including participant demographics. Three studies investigated a higher percentage of women than men, with only Remvig and colleagues [14] not detailing the sex of participants. One study excluded men altogether [16], stating that $90 \%$ of hEDS patients are women. Most studies recruited convenience samples from existing clinical records, with only Alsiri and colleagues [15] recruiting prospectively from physiotherapy referrals.

Age ranges varied from 18 to 89 years, which is an important factor when measuring the mechanical properties of skin, because of collagen changes with age. For example, Luebberding and colleagues [22] identified greater laxity and reduced elasticity in skin after 40 years of age. An additional relationship was observed between reduced skin elasticity in women and hormonal changes over time, particularly during the menopause. These observations are important because all four studies in this review included women over 40 years (although Remvig and colleagues [14] capped the 
Fig. 1 PRISMA flow diagram [19] illustrating the process of searching and selecting the studies included in the review

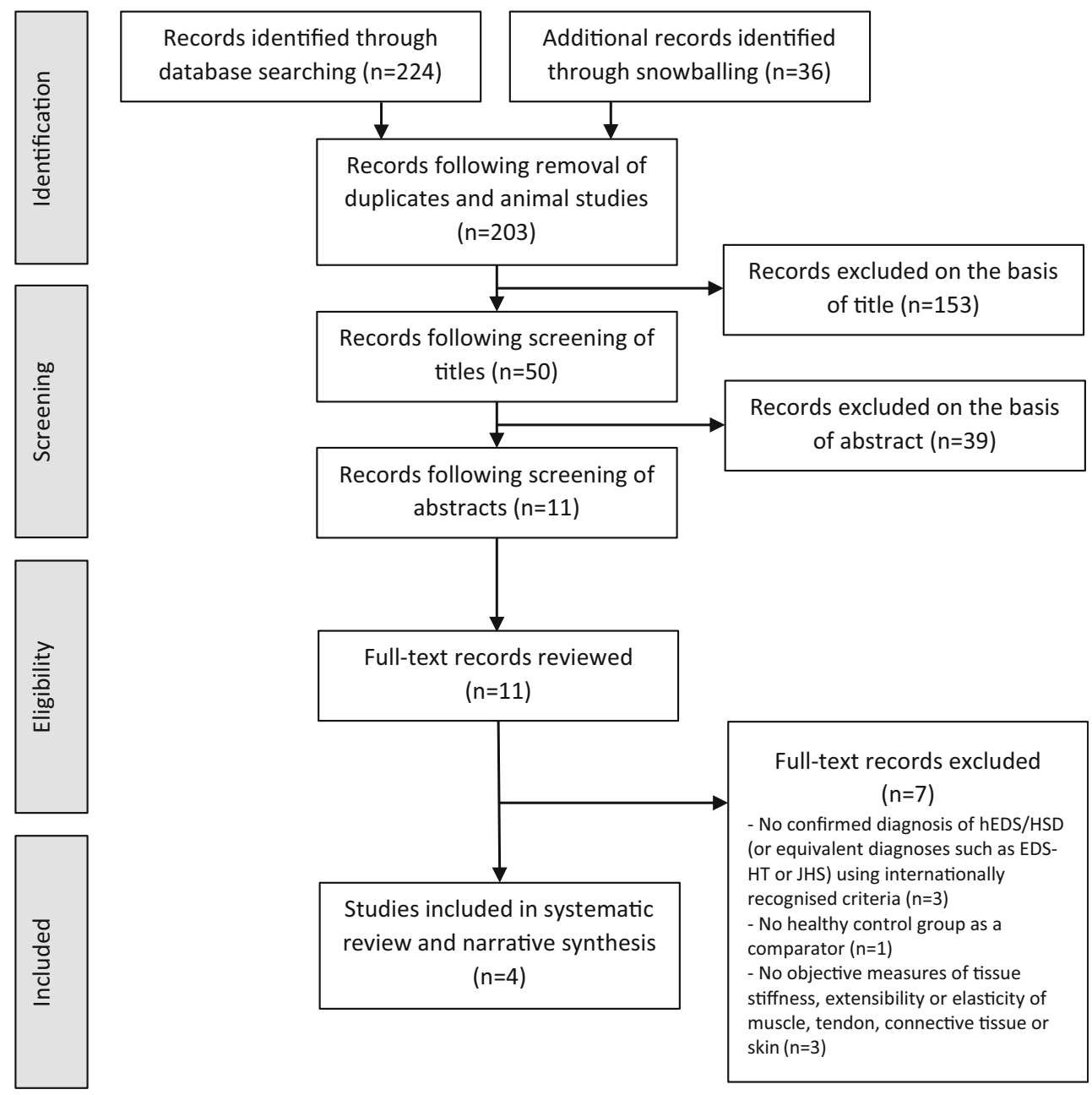

age at 42 citing age-related collagen changes for this decision). Age could therefore be a confounding factor for each of the studies and further research should be conducted to understand its impact on the data.

Reporting of ethnicity can improve judgements of external validity, particularly as to whether the sample represents the diversity of affected populations [23]. Ethnicity is particularly important in this case because hypermobility is known to be more prevalent in African and Asian groups as compared to white populations [24]. However, ethnicity of participants was reported in only one study [15].

\section{Study quality}

The STROBE statement (Table 3) identified that overall there was robust reporting and analysis of results, including declarations of limitations and/or recommendations for future practice and research. Omissions in some reports related to criteria 5, 9 and 10. Only Alsiri and colleagues [15] provided robust details for criterion 5 ('Describe the setting, locations and relevant dates, including periods of recruitment, exposure, follow-up and data collection'). Two studies $[14,15]$ supplied details for criterion 9 ('Describe any efforts to address potential sources of bias'). Only one study [15] provided evidence for criterion 10 ('Explain how the study size was arrived at'). This demonstrates that the common omissions are related to time scales and recruitment processes, blinding to reduce bias and sample size calculations. Alsiri and colleagues [15] was the only study to meet all STROBE criteria, indicating a highquality study report.

Details about the recruitment process are important as they provide context for the reader and clarity about whether participants are suitable for answering the study objectives. Only Alsiri and colleagues [15] provided sufficient detail about the study setting, including such details as the dates during which data collection was conducted.

Blinding is the most effective way of preventing investigator effects or expectation effects but there was a lack of blinding in two of the four studies [16, 21]. It should be noted that blinding was incomplete in both of the studies that attempted it $[14,15]$. For example, in Alsiri and colleagues [15], the examiner knew the diagnostic category of each participant and blinding only applied to data analysis. Remvig and colleagues [14] reported blinding examiners to previous 


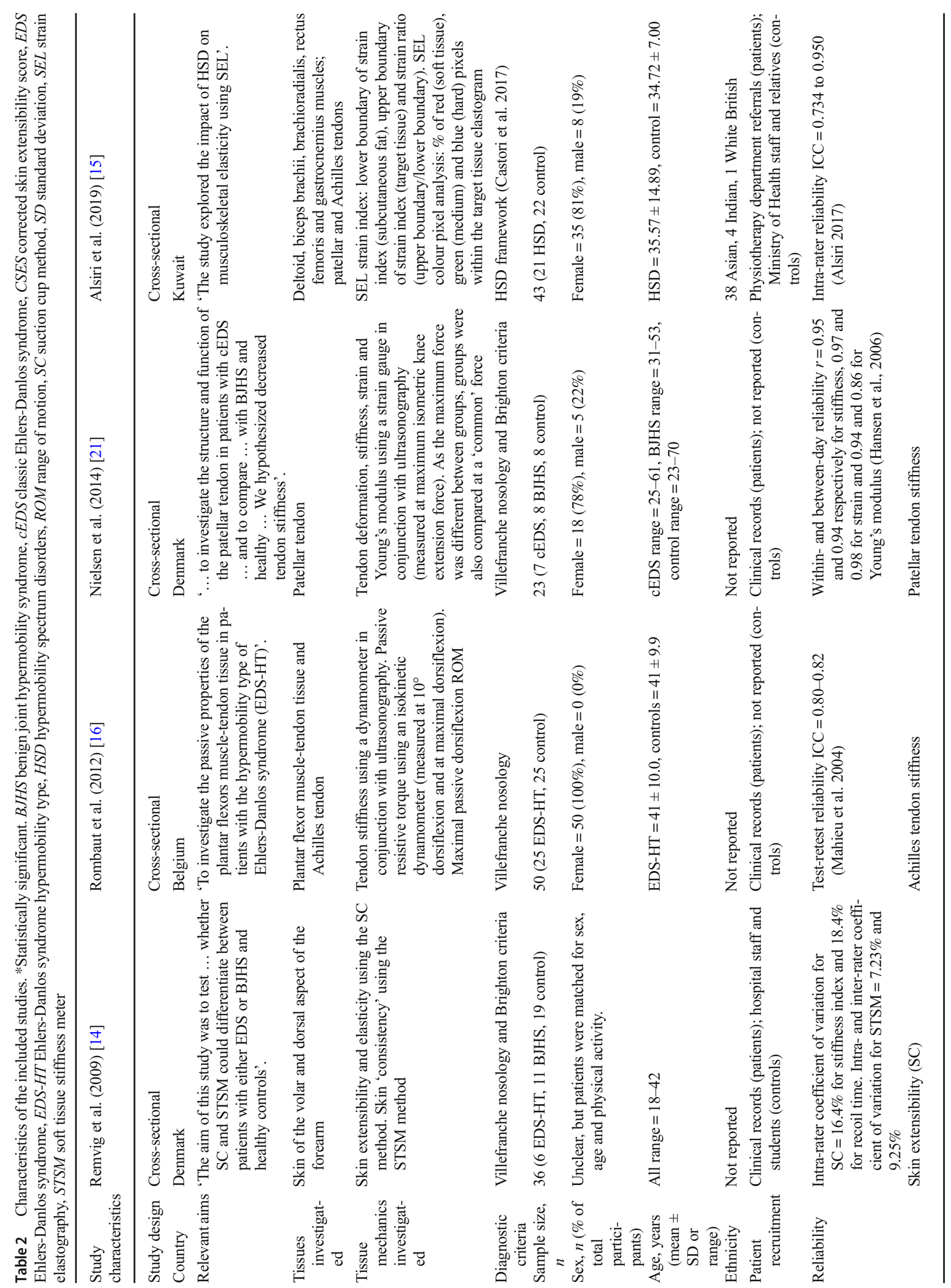




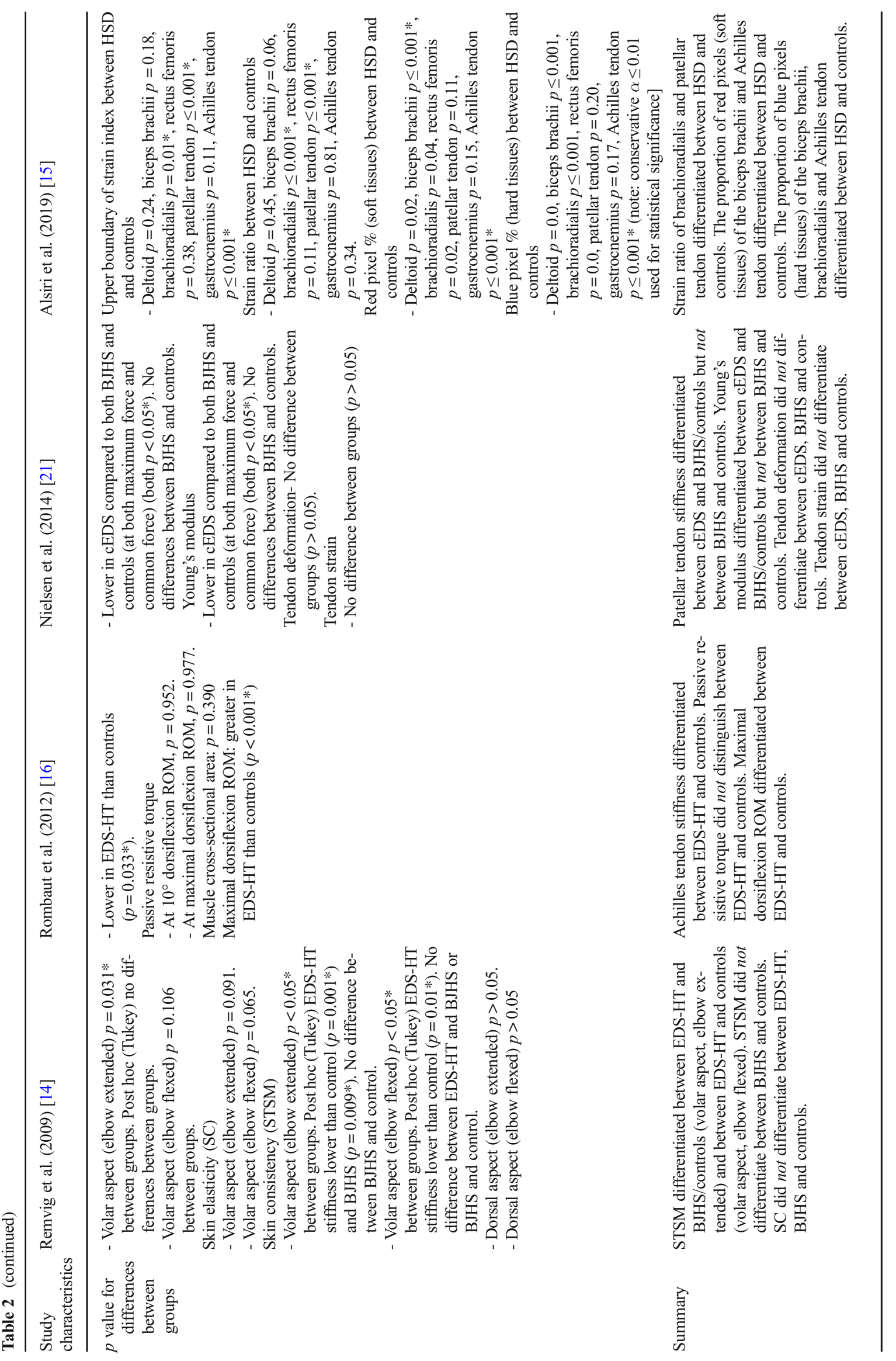


Table 3 STROBE checklist for cross-sectional studies [20]. * Information must be provided separately for each group; $\checkmark$ information was provided or an explanation for its absence was given; $\mathrm{X}$ information was absent and no explanation for this was given

\begin{tabular}{|c|c|c|c|c|}
\hline STROBE criteria & $\begin{array}{l}\text { Remvig } \\
\text { et al. [14] }\end{array}$ & $\begin{array}{l}\text { Rombaut } \\
\text { et al. [16] }\end{array}$ & $\begin{array}{l}\text { Nielsen } \\
\text { et al. [21] }\end{array}$ & $\begin{array}{l}\text { Alsiri } \\
\text { et al. [15] }\end{array}$ \\
\hline
\end{tabular}

Title and abstract

1 a). Indicate the study's design with a commonly used term in the title or abstract

b). Provide in the abstract an informative and balanced summary of what was done and what was found

Introduction

Background/rationale

2. Explain the scientific background and rationale for the investigation being reported Objectives

3. State-specific objectives, including any pre-specified hypotheses

Methods

Study design

4. Present key elements of study design early in the paper

Setting

5. Describe the setting, locations and relevant dates, including periods of recruitment, exposure, follow-up and data collection

Participants

6. Give the eligibility criteria, and the sources and methods of selection participants

Variables

7. Clearly define all the outcomes, exposures, predictors, potential confounders, and effect modifiers. Give diagnostic criteria, if applicable

Data sources/ measurement

$8^{*}$. For each variable of interest, give sources of data and details of methods of assessment (measurement) Describe comparability of assessment methods if there is more than one Bias group

9. Describe any efforts to address potential sources of bias

Study size

10. Explain how the study size was arrived at

Quantitative variables

11. Explain how quantitative variables were handled in the analysis. If applicable describe which groupings were chosen and why

Statistical methods

12 a). Describe all statistical methods, including those used to control for confounding

b). Describe any methods used to examine subgroups and interactions

c). Explain how missing data were addressed

d). If applicable, describe analytical methods taking account of sampling strategy

e). Describe any sensitivity analyses

Results

Participants

$13^{*}$ a). Report numbers of individuals at each stage of the study

b). Give reasons for non-participation at each stage

Descriptive data

14 a). Give characteristics of participants

b). Indicate number of participants with missing data

Outcome data

$15^{*}$. Report numbers of outcome events or summary measures

Main results

16 a). Give unadjusted data, adjusted data and reasons for adjustments

b). Report category boundaries when continuous variables were categorised

\begin{tabular}{|c|c|c|}
\hline$\checkmark$ & $\mathrm{X}$ & $X$ \\
\hline$X$ & $X$ & $X$ \\
\hline$\checkmark$ & $\checkmark$ & $\checkmark$ \\
\hline$\checkmark$ & $\checkmark$ & $\checkmark$ \\
\hline$\checkmark$ & $\checkmark$ & $\checkmark$ \\
\hline$\checkmark$ & $\checkmark$ & $\checkmark$ \\
\hline$\checkmark$ & $\checkmark$ & $\checkmark$ \\
\hline$\checkmark$ & $\checkmark$ & $\checkmark$ \\
\hline
\end{tabular}


Table 3 (continued)

\begin{tabular}{|c|c|c|c|c|}
\hline STROBE criteria & $\begin{array}{l}\text { Remvig } \\
\text { et al. [14] }\end{array}$ & $\begin{array}{l}\text { Rombaut } \\
\text { et al. [16] }\end{array}$ & $\begin{array}{l}\text { Nielsen } \\
\text { et al. [21] }\end{array}$ & $\begin{array}{l}\text { Alsiri } \\
\text { et al. [15] }\end{array}$ \\
\hline \multicolumn{5}{|l|}{ Other analyses } \\
\hline 17. Report other analyses done e.g. subgroups and sensitivity analyses & $\checkmark$ & $\checkmark$ & $\checkmark$ & $\checkmark$ \\
\hline \multicolumn{5}{|l|}{ Discussion } \\
\hline \multicolumn{5}{|l|}{ Key results } \\
\hline 18. Summarise key results with reference to study objectives & $\checkmark$ & $\checkmark$ & $\checkmark$ & $\checkmark$ \\
\hline \multicolumn{5}{|l|}{ Limitations } \\
\hline $\begin{array}{l}\text { 19. Discuss limitations of the study, including bias and imprecision. Discuss direction and } \\
\text { magnitude of bias }\end{array}$ & $\checkmark$ & $\checkmark$ & $\mathrm{X}$ & $\checkmark$ \\
\hline \multicolumn{5}{|l|}{ Interpretation } \\
\hline $\begin{array}{l}\text { 20. Give a cautious overall interpretation of results considering objectives, limitations, } \\
\text { multiplicity of analyses, results from similar studies and other evidence }\end{array}$ & $\checkmark$ & $\checkmark$ & $\checkmark$ & $\checkmark$ \\
\hline \multicolumn{5}{|l|}{ Generalisability } \\
\hline 21. Discuss the generalisability (external validity) of the study results & $\checkmark$ & $\checkmark$ & $\checkmark$ & $\checkmark$ \\
\hline \multicolumn{5}{|l|}{ Other information } \\
\hline \multicolumn{5}{|l|}{ Funding } \\
\hline 22. Give the source of any funding and their role in this study or previous studies & $\checkmark$ & $\mathrm{X}$ & $\checkmark$ & $\checkmark$ \\
\hline
\end{tabular}

skin signs and to each other's results but it was not reported if the examiners were aware of specific diagnoses or if analysis was conducted blind.

Sample size calculations allow researchers to generalise results and it has been suggested that a sample size calculation should be a prerequisite to any clinical research study [25]. The study by Alsiri and colleagues [15] was the only one to conduct an a priori sample size calculation. They used data from a previous $\mathrm{PhD}$ study [26] to guide the calculation, identifying that 20 participants were needed. They proceeded to recruit and retain appropriate numbers to fulfil the calculated numbers. As the sample size of the other three studies was not justified, the results may have been subject to type II errors.

All studies had robust inclusion and exclusion criteria which indicated attempts to mitigate potential confounding factors. Exclusion criteria included skin disease, malignancies and limb trauma, all of which could affect skin/tissue mechanics. Pregnancy and recent childbirth were exclusions employed by Alsiri and colleagues [15] and Rombaut and colleagues [16]. An increase of relaxin levels during pregnancy, and shortly after birth, is associated with increased joint laxity. Relaxin regulates the remodelling of tissue structures such as bone, synovium, cartilage, ligaments and tendons [27]. Exclusion of pregnant women, and those that have recently given birth, therefore attempts to reduce the risk of this confounding factor. However, there is also evidence that increased relaxin levels during menstruation in normally menstruating young women were associated with a reduction in patellar tendon stiffness [28]. The role of relaxin on tissue stiffness in women with hEDS/HSD may therefore be important to consider in future research [16].

\section{Assessment methods}

There were a variety of methods shown to distinguish between the tissue mechanics of adults with hEDS/HSD and healthy controls. These methods were applied to a range of different tissues. The following discussion evaluates the potential clinical utility of such methods.

Remvig and colleagues [14] used two established techniques for measuring skin extensibility, elasticity and stiffness - the suction cup method (SC) and the soft tissue stiffness meter (STSM). They explored if the devices could differentiate between EDS-HT, BJHS and controls. The study identified a difference between the three groups in skin extensibility of the volar aspect of the forearm using the SC method when the arm was extended but not flexed. However, on post hoc testing, there were no differences between individual groups. There were no differences in skin elasticity between groups using the SC method. The STSM found differences in skin consistency on the volar aspect of the forearm between groups with the elbow both extended and flexed. People with EDS-HT had lower stiffness than controls but there were no differences between BJHS and control. There were no differences in skin consistency as measured with the STSM on the dorsal aspect of the forearm. Arokoski and colleagues [29] previously demonstrated the feasibility of the custom-built STSM used in the study. Other soft tissue meters are now commercially available, are practical and have generally been evaluated positively [30, 31]. Further evaluation of such devices in the assessment of hEDS/HSD is recommended. Given the observation that differences were observed in the volar aspect of the forearm but not the dorsal aspect [14], a range of anatomical structures should be tested in future research. 
Rombaut and colleagues [16] investigated the mechanical properties of the plantar flexor muscle-tendon tissues in people with EDS-HT and controls. Achilles tendon stiffness was measured as a function of tendon lengthening (assessed using diagnostic ultrasound) and isometric plantar flexor muscle force (assessed using an isokinetic dynamometer). Stiffness of the Achilles tendon was significantly reduced in adults with EDS-HT (EDS-HT $12.3 \pm 4.84 \mathrm{~N} / \mathrm{mm}$ versus controls $15.6 \pm$ 4.43). The clinical utility of such methods may be more challenging due to the specialist equipment and training required.

Alsiri and colleagues [15] used strain elastography to examine passive stiffness in the Achilles tendon, patellar tendon and five muscles. The technique involves pressing an ultrasound probe rhythmically over the site of interest and the digital capture of the ultrasound image (an elastogram) of the compression of the underlying tissues (strain). Analysis involved highlighting an area of the elastogram which represented the area of interest and another reference area of subcutaneous fat. A strain index was automatically calculated for each area, with the upper boundary representing the area of interest and lower boundary representing the subcutaneous fat. From this, the strain ratio (upper boundary/lower boundary) was calculated. The elastograms are also colour coded, producing a map of soft (coloured red), intermittent (green) and hard tissues (blue). Alsiri and colleagues [15] calculated the proportion of red, green and blue pixels within each target tissue using an image processing software. The upper boundary of the strain index successfully distinguished between people with HSD and healthy controls at three test sites: brachioradialis muscle, patellar tendon and Achilles tendon. The strain ratio distinguished between groups at two test sites: brachioradialis muscle and patellar tendon. There was a higher proportion of soft tissues in the biceps brachii and Achilles tendon and a lower proportion of hard tissues in the biceps brachii, brachioradialis and Achilles tendon in people with HSD, when compared to healthy controls. Of these sites, the Achilles tendon seemed particularly promising as differences were observed using three outcomes - the strain index and the proportion of both soft and hard tissues. Although elastography seems to be becoming more popular, with an increasing evidence base, its clinical utility might still be questioned due to the specialist equipment and training required. Nevertheless, it is clearly sensitive to soft tissue changes in hEDS/HSD and warrants further investigation. An alternative to strain elastography, namely shear wave elastography, has the potential to provide even more objective findings [32] and could form the basis of future research.

\section{Strengths and limitations}

The design and conduct of the review were extremely rigorous, with all steps of the procedure subject to multiple researcher oversight, discussion and consensus. The review was limited to the English language, however, and may have excluded relevant studies as a result. Heterogeneity of diagnostic criteria and terminology made it difficult to identify the patient populations investigated, although we are confident that the studies included were appropriate to the inclusion criteria.

\section{Recommendations for future research}

Future research should further investigate the potential use of handheld tissue stiffness meters and ultrasound methods to contribute towards the diagnosis of hEDS/HSD. The former devices may be particularly useful in clinical practice as they require minimal training. Appropriate clinimetric properties, such as sensitivity and specificity and receiver operating curve analysis, should be explored. In the absence of a gold standard diagnostic test, comparisons should be made against the international diagnostic criteria for hEDS [1] and framework for HSD [2]. Additionally, it is recommended that attention is paid to recruiting representative samples in terms of age, gender and ethnicity. Future studies should include blinding to avoid investigator bias and should perform prospective study size calculations.

\section{Conclusion}

In conclusion, a limited range of studies were available to address the review's aim. Three of the four studies included reported at least one measure of tissue mechanics that distinguished between adults with hEDS/HSD and healthy participants. These included assessment of skin, muscle (brachioradialis and biceps brachii) and tendon (patellar and Achilles). Assessment methods included the soft tissue stiffness meter and diagnostic ultrasound, including strain elastography. The assessment methods seem clinically practical but require further validation, particularly within diagnostic scenarios.

Acknowledgements The authors would like to acknowledge the assistance of Pauline Shaw, subject librarian at the University of the West of England, Bristol.

\section{Compliance with ethical standards}

Disclosures None.

Open Access This article is licensed under a Creative Commons Attribution 4.0 International License, which permits use, sharing, adaptation, distribution and reproduction in any medium or format, as long as you give appropriate credit to the original author(s) and the source, provide a link to the Creative Commons licence, and indicate if changes were made. The images or other third party material in this article are included in the article's Creative Commons licence, unless indicated otherwise in a credit line to the material. If material is not included in the article's 
Creative Commons licence and your intended use is not permitted by statutory regulation or exceeds the permitted use, you will need to obtain permission directly from the copyright holder. To view a copy of this licence, visit http://creativecommons.org/licenses/by/4.0/.

\section{References}

1. Malfait F, Francomano C, Byers P, Belmont J, Berglund B, Black J, Bloom L, Bowen JM, Brady AF, Burrows NP, Castori M, Cohen H, Colombi M, Demirdas S, De Backer J, De Paepe A, FournelGigleux S, Frank M, Ghali N, Giunta C, Grahame R, Hakim A, Jeunemaitre X, Johnson D, Juul-Kristensen B, Kapferer-Seebacher I, Kazkaz H, Kosho T, Lavallee ME, Levy H, Mendoza-Londono R, Pepin M, Pope FM, Reinstein E, Robert L, Rohrbach M, Sanders L, Sobey GJ, Van Damme T, Vandersteen A, van Mourik C, Voermans N, Wheeldon N, Zschocke J, Tinkle B (2017) The 2017 international classification of the Ehlers-Danlos syndromes. Am J Med Genet C: Semin Med Genet 175(1):8-26. https://doi.org/ 10.1002/ajmg.c.31552

2. Castori M, Tinkle B, Levy H, Grahame R, Malfait F, Hakim A (2017) A framework for the classification of joint hypermobility syndrome and related conditions. Am J Med Genet C: Semin Med Genet 175(1):148-157. https://doi.org/10.1002/ajmg.c.31539

3. Tinkle B, Castori M, Berglund B, Cohen H, Grahame R, Kazkaz H, Levy H (2017) Hypermobile Ehlers-Danlos syndrome (a.k.a. Ehlers-Danlos syndrome type III and Ehlers-Danlos syndrome hypermobility type): clinical description and natural history. Am J Med Genet Part C Semin Med Genet 175C:48-69. https://doi.org/ 10.1002/ajmg.c.31538

4. Chan C, Krahe A, Lee YT, Nicholson LL (2019) Prevalence and frequency of self-perceived systemic features in people with joint hypermobility syndrome/Ehlers-Danlos syndrome hypermobility type. Clin Rheumatol 38:503-511. https://doi.org/10.1007/ s10067-018-4296-7

5. Russek LN, Errico DM (2016) Prevalence, injury rate and, symptom frequency in generalized joint laxity and joint hypermobility syndrome in a "healthy" college population. Clin Rheumatol 35(4): 1029-1039. https://doi.org/10.1007/s10067-015-2951-9

6. Albayrak İ, Yilmaz H, Akkurt HE, Salli A, Karaca G (2015) Is pain the only symptom in patients with benign joint hypermobility syndrome? Clin Rheumatol 34:1613-1619. https://doi.org/10.1007/ s10067-014-2610-6

7. Beighton P, De Paepe A, Steinmann B, Tsipouras P, Wenstrup RJ (1998) Ehlers-Danlos syndromes: revised nosology, Villefranche, 1997. Am J Med Genet 77:31-37. https://doi.org/10.1002/(sici) 1096-8628(19980428)77:1<31::aid-ajmg8>3.0.co;2-o

8. Grahame R, Bird HA, Child A (2000) The revised (Brighton 1998) criteria for the diagnosis of benign joint hypermobility syndrome (BJHS). J Rheumatol 27(7):1777-1779

9. Palmer S, Bailey S, Barker L, Barney L, Elliott A (2014) The effectiveness of therapeutic exercise for joint hypermobility syndrome: a systematic review. Physiotherapy 100:220-227. https:// doi.org/10.1016/j.physio.2013.09.002

10. Beighton P, Solomon L, Soskolne CL (1973) Articular mobility in an African population. Ann Rheum Dis 32:413-418. https://doi. org/10.1136/\%2Fard.32.5.413

11. Palmer S, Cramp F, Lewis R, Gould G, Clark EM (2017) Development and initial validation of the Bristol Impact of Hypermobility questionnaire. Physiotherapy. 103(2):186-192. https://doi.org/10.1016/j.physio.2016.04.002
12. Day H, Koutedakis Y, Wyon MA (2011) Hypermobility and dance: a review. Int J Sports Med 32(7):485-489. https://doi.org/10.1055/ s-0031-1273690

13. Juul-Kristensen B, Schmedling K, Rombaut L, Lund H, Engelbert RH (2017) Measurement properties of clinical assessment methods for classifying generalized joint hypermobility - a systematic review. Am J Med Genet C: Semin Med Genet 175(1):116-147. https://doi.org/10.1002/ajmg.c.31540

14. Remvig L, Duhn PH, Ullman S, Kobayasi T, Hansen B, JuulKristensen B, Jurvelin JS, Arokoski J (2009) Skin extensibility and consistency in patients with Ehlers-Danlos syndrome and benign joint hypermobility syndrome. Scand J Rheumatol 38(3):227230. https://doi.org/10.1080/03009740802520714

15. Alsiri N, Al-Obaidi S, Asbeutah A, Almandeel M, Palmer S (2019) The impact of hypermobility spectrum disorders on musculoskeletal tissue stiffness: an exploration using strain elastography. Clin Rheumatol 38(1):85-95. https://doi.org/10.1007/s10067-0184193-0

16. Rombaut L, Malfait F, De Wandele I, Mahieu N, Thijs Y, Segers P, De Paepe A, Calders P (2012) Muscle-tendon tissue properties in the hypermobility type of Ehlers-Danlos syndrome. Arthritis Care Res 64(5):766-772. https://doi.org/10.1002/acr.21592

17. Heidbreder AE, Ringelstein EB, Dittrich R, Nabavi D, Metze D, Kuhlenbaäumer G (2008) Assessment of skin extensibility and joint hypermobility in patients with spontaneous cervical artery dissection and Ehlers-Danlos syndrome. J Clin Neurosci 15:650-653. https://doi.org/10.1016/j.jocn.2007.08.001

18. Grahame R, Beighton P (1969) Physical properties of the skin in the Ehlers-Danlos syndrome. Ann Rheum Dis 28:246-251. https://doi. org/10.1136/ard.28.3.246

19. Moher D, Liberati A, Tetzlaff J, Altman DG, PRISMA Group (2009) Preferred reporting items for systematic reviews and metaanalyses: the PRISMA statement. PLoS Med 6(7):e1000097. https://doi.org/10.1371/journal.pmed.1000097

20. von Elm E, Altman DG, Egger M, Pocock SJ, Gøtzsche PC, Vandenbroucke JP, Initiative STROBE (2008) The Strengthening the Reporting of Observational Studies in Epidemiology (STROBE) statement: guidelines for reporting observational studies. J Clin Epidemiol 61(4):344-349. https://doi.org/10.1016/j. jclinepi.2007.11.008

21. Nielsen RH, Couppé C, Jensen JK, Olsen MR, Heinemeier KM, Malfait F, Symoens S, De Paepe A, Schjerling P, Magnusson SP, Remvig L, Kjaer M (2014) Low tendon stiffness and abnormal ultrastructure distinguish classic Ehlers-Danlos syndrome from benign joint hypermobility syndrome in patients. FASEB J 28(11): 4668-4676. https://doi.org/10.1096/fj.14-249656

22. Luebberding S, Krueger N, Kerscher M (2014) Mechanical properties of human skin in vivo: a comparative evaluation in 300 men and women. Skin Res Technol 20(2):127-135. https://doi.org/10. 1111/srt.12094

23. Hyllegard R, Mood DP, Morrow JR (1996) Research validity. In: Allman KL (ed) Interpreting research in sports and exercise science. WCB McGraw-Hill, London, pp 135-144

24. Hakim A, Grahame R (2003) Joint hypermobility. Best Pract Res Clin Rheumatol 17(6):989-1004. https://doi.org/10.1016/j.berh. 2003.08.001

25. Nayak BK (2010) Understanding the relevance of sample size calculation. Indian J Ophthalmol 58(6):469-470. https://doi.org/10. 4103/\%2F0301-4738.71673

26. Alsiri NF (2017) The impact of joint hypermobility syndrome in adults: a quantitative exploration of neuromuscular impairments, activity limitations and participation restrictions. $\mathrm{PhD}$ thesis, 
University of the West of England, Bristol, UK. http://eprints.uwe. ac.uk/30112/18/Thesis_Najla_4RegistoryV2.pdf

27. Dehghan F, Haerian BS, Muniandy S, Yusof A, Dragoo JL, Salleh N (2014) The effect of relaxin on the musculoskeletal system. Scand J Med Sci Sports 24:e220-e229. https://doi.org/10.1111/ sms. 12149

28. Pearson SJ, Burgess KE, Onambélé GL (2011) Serum relaxin levels affect the in vivo properties of some but not all tendons in normally menstruating young women. Exp Physiol 96(7):681-688. https:// doi.org/10.1113/expphysiol.2011.057877

29. Arokoski JP, Surakka J, Ojala T, Kolari P, Jurvelin JS (2005) Feasibility of the use of a novel soft tissue stiffness meter. Physiol Meas 26(3):215-228. https://doi.org/10.1088/0967-3334/26/3/007

30. Chino K, Takahashi H (2016) Handheld tissue hardness meters for assessing the mechanical properties of skeletal muscle: a feasibility study. J Manip Physiol Ther 39(7):518-522. https://doi.org/10. 1016/j.jmpt.2016.07.002

31. Feng YN, Li YP, Liu CL, Zhang ZJ (2018) Assessing the elastic properties of skeletal muscle and tendon using shearwave ultrasound elastography and MyotonPRO. Sci Rep 8(1):17064. https:// doi.org/10.1038/s41598-018-34719-7

32. Washburn N, Onishi K, Wang JH-C (2018) Ultrasound elastography and ultrasound tissue characterisation for tendon evaluation. J Orthop Translat 15:9-20. https://doi.org/10.1016/j.jot. 2018.06.003

Publisher's note Springer Nature remains neutral with regard to jurisdictional claims in published maps and institutional affiliations. 